\title{
A STRONG CONTAINMENT PROPERTY FOR DISCRETE AMENABLE GROUPS OF AUTOMORPHISMS ON $W^{*}$ ALGEBRAS
}

\author{
EDMOND E. GRANIRER
}

\author{
This paper is dedicated with affection and gratitude to \\ my father Dr. Jacob Granirer
}

\begin{abstract}
Let $G$ be a countable group of automorphisms on a $W^{*}$ algebra $\mathcal{M}$ and let $\phi_{0}$ be a $w^{*} G_{\delta}$ point of the set of $G$ invariant states on $\mathcal{M}$ which belong to $w^{*} \operatorname{cl} \operatorname{Co} E$, where $E$ is a set of (possibly pure) states on $\mathcal{M}$. If $G$ is amenable, then the cyclic representation $\pi_{\phi_{0}}$ corresponding to $\phi_{0}$ is contained in $\left\{\bigoplus \pi_{\phi} ; \phi \in E\right\}$. This property characterizes amenable groups. Related results are obtained.
\end{abstract}

Introduction. Let $\mathcal{M}$ be an infinite dimensional $W^{*}$ algebra, $\mathcal{M}^{*}$ its Banach space dual, $G$ a group of automorphisms $g: \mathcal{M} \rightarrow \mathcal{M}$, and $E$ a set of states on $\mathcal{M}$ such that $G^{*} E \subset E$. Denote by $S_{E}^{G}$ the set of states $\phi$ in $w^{*} \operatorname{cl} \operatorname{Co} E$ (the $w^{*}$ closure of the convex hull of $E$ ) which are $G$-invariant, i.e. $\phi(g x)=\phi(x)$ for all $g$ in $G$ and $x$ in $\mathcal{M}$.

We call $\phi_{0}$ in $S_{E}^{G}$ a $w^{*} G_{\delta}$ point of $S_{E}^{G}$ if there is a sequence $J=\left\{x_{n}\right\}$ in $\mathcal{M}$ such that $\left\{\phi_{0}\right\}=S_{E}^{G} \cap J^{0}$, where $J^{0}=\left\{\phi \in \mathcal{M}^{*} ;\langle\phi, J\rangle=0\right\}$. This just means that the $w^{*}$ topology restricted to $S_{E}^{G}$ is first countable at $\phi_{0}$. Denote by $w^{*} G_{\delta}\left(S_{E}^{G}\right)$ the set of all such points. We note that $\phi_{0}$ being in $w^{*} G_{\delta}\left(S_{E}^{G}\right)$ depends only on the $w^{*}$ topology restricted to $S_{E}^{G}$ and does not depend a priori, on what happens in $w^{*} \operatorname{cl~Co} E$. Furthermore $w^{*} G_{\delta}\left(S_{E}^{G}\right)$ may be void. If for some countable $J \subset \mathcal{M}$ the set $\left(S_{E}^{G} \cap J^{0}, w^{*}\right)$ is separable metric or even if it has the RNP (even the WRNP is enough in some cases; see the sequel for notations), then $w^{*} G_{\delta}\left(S_{E}^{G}\right) \neq \varnothing$.

If $\phi \in \mathcal{M}^{*}$ is positive let $\pi_{\phi}$ be the GNS representation determined by $\phi$ (Pedersen $[12,3.3 .3])$.

The main result of this paper is (a refinement of) the following

THEOREM. Let $\phi_{0} \in w^{*} G_{\delta}\left(S_{E}^{G}\right)$. Then $\pi_{\phi_{0}}$ is contained (not only weakly contained à la Fell) in the direct sum $\left\{\bigoplus \pi_{\phi} ; \phi \in E\right\}$ provided $G$ is countable and amenable.

If $G$ is any nonamenable group then there is even an abelian $\mathcal{M}=L^{\infty}(X \mu)$ for some nonatomic probability space $(X, B, \mu)$ on which $G$ acts ergodically and measure preservingly such that if $E$ is the set of all pure states on $\mathcal{M}$ then $w^{*} G_{\delta}\left(S_{E}^{G}\right)=$ $\{\mu\}=S_{E}^{G}$, yet $\pi_{\mu}$ is not contained (but only weakly contained) in $\left\{\bigoplus \pi_{\phi} ; \phi \in E\right\}$.

Received by the editors November 4, 1985.

1980 Mathematics Subject Classification (1985 Revision). Primary 46L30, 46L40, 46L55; Secondary 46L10. 
Several corollaries of this result follow and the unifying thread of them all is that they characterize the amenability of $G$ in the class of countable groups. Furthermore they generalize to the noncommutative case our results in [9].

Corollary 1 deals with the case where $E$ consists of pure states on $\mathcal{M}$. We show that in this case the "support" of any such $\phi_{0} \in w^{*} G_{\delta}\left(S_{E}^{G}\right)$ is a countable set $R \subset \hat{\mathcal{M}}$ (the irreducible representations of $\mathcal{M}$ modulo unitary equivalence) such that $R=\bigcup R_{k}$, a disjoint union of finite $G$ invariant subsets $R_{k}$, provided $G$ is countable and amenable. Again in the absence of amenability this " $w^{*} G_{\delta}$-finite invariant" property of points in $w^{*} G_{\delta}\left(S_{E}^{G}\right)$ fails (even for abelian $\mathcal{M}$ ). This corollary is a generalization to the nonabelian case of (an improved version of) our results in [9].

The remainder of the corollaries deal with the case that $S_{E}^{G} \cap J^{0}$ has the RNP (or $S_{E}^{G}$ has the WRNP under the additional assumption that $S^{G}$ is a simplex). In this case $w^{*} G_{\delta}\left(S_{E}^{G}\right) \neq \varnothing$.

One of the main tools in the proofs is the " $w^{*} G_{\delta}$ sequential property" of countable (left) amenable semigroups introduced in [9], a property which characterizes amenability in the class of countable groups. As shown in [18, pp. 47-48] there are noncountable abelian (a fortiori amenable) groups which do not possess this property.

In the end we point out that in case $E \subset \mathcal{M}_{*}$ consists of normal states better results are available and the amenability of $G$ does not come into play.

Definitions and notations. $\mathcal{M}$ will always denote an infinite dimensional $W^{*}$ algebra, $\mathcal{M}^{*}$ its Banach space dual (for the unexplained notations we follow Pedersen [12]), and $S_{\mathcal{M}}=S \subset \mathcal{M}^{*}$ the set of states. If $E \subset \mathcal{M}^{*}$, let $\operatorname{Co} E$ denote the convex hull of $E$ and $w^{*} \operatorname{cl} \operatorname{Co} E$ the $w^{*}=\sigma\left(\mathcal{M}^{*}, \mathcal{M}\right)$ closure of Co $E$. (If $X, Y$ are linear spaces in duality, $\sigma(X, Y)$ is the weakest topology on $X$ which renders all linear functions in $Y$ continuous.) $\mathcal{M}_{*}$ denotes the predual of $\mathcal{M}$.

If $\phi$ is a positive element in $\mathcal{M}^{*}$, then $\left(\pi_{\phi}, H_{\phi}, h_{\phi}\right)$ will denote the (GNS) cyclic representation induced by $\phi[12,3.3 .3]$. It acts on the Hilbert space $H_{\phi}$ and is such that $\phi(x)=\left\langle\pi_{\phi}(x) h_{\phi}, h_{\phi}\right\rangle$, where $h_{\phi} \in H_{\phi}$ is the cyclic vector.

Let Aut $\mathcal{M}$ be the set of all automorphisms of $\mathcal{M}$. Let $G \subset$ Aut $\mathcal{M}$ be a semigroup. If $g \in G$ let $g^{*}: \mathcal{M}^{*} \rightarrow \mathcal{M}^{*}$ be defined by $\left(g^{*} \phi\right)(x)=\phi(g x)$ for all $x$ in $\mathcal{M}$.

If $E \subset \mathcal{M}^{*}$ let $G^{*} E=\left\{g^{*} \phi ; g \in G, \phi \in E\right\} . G^{*} \phi=\phi$ will just mean that $g^{*} \phi=\phi$ for all $g$ in $G$. If $E \subset S$ let $S_{E}^{G}=\left\{\phi \in w^{*} \operatorname{clCo} E ; G^{*} \phi=\phi\right\}$ and $S^{G}=\left\{\phi \in S ; G^{*} \phi=\phi\right\}$ the set of all $G$ invariant states.

If $K \subset S$ then $\phi_{0}$ is a $w^{*} G_{\delta}$ point of $K$ if there are $x_{n}$ in $\mathcal{M}$ and scalars $\alpha_{n}, n=1,2,3, \ldots$, depending on $\phi_{0}$, such that $\left\{\phi_{0}\right\}=\left\{\phi \in K ; \phi\left(x_{n}\right)=\alpha_{n}, n \geq\right.$ 1\}. $w^{*} G_{\delta}(K)$ denotes the set of all such $\phi$ in $K$. Since $\phi(I)=1$ for all $\phi$ in $S$, $\phi_{0} \in w^{*} G_{\delta}(K)$ iff there is a separable subspace $J \subset \mathcal{M}$ such that $J^{0} \cap K=\left\{\phi_{0}\right\}$, where $J^{0}=\left\{\phi \in \mathcal{M}^{*} ; \phi(x)=0\right.$ for all $x$ in $\left.J\right\}$.

$\hat{\mathcal{M}}$ will denote the set of all irreducible unitary representations of $\mathcal{M}$ modulo unitary (spatial in $[\mathbf{1 2}, 3.3 .6]$ ) equivalence.

If $\left(\pi_{1}, H_{1}\right),\left(\pi_{2}, H_{2}\right)$ are unitary representations of $\mathcal{M}[12,3.3 .1]$ we write $\pi_{1} \leq$ $\pi_{2}$ if $\pi_{1}$ is unitarily equivalent to a subrepresentation of $\pi_{2}$. If $g \in$ Aut $\mathcal{M}$ and $(\pi, H)$ a representation of $\mathcal{M}$, then $\hat{g} \pi$ is the representation of $\mathcal{M}$ on $H$ given by $(\hat{g} \pi)(x)=\pi(g x)$ i.e. $g \pi=\pi \circ g$. Note that if $\left(\pi_{1} H_{1}\right),\left(\pi_{2} H_{2}\right)$ are unitarily equivalent representations (denoted by $\sim$ ), i.e. for some unitary $u: H_{1} \rightarrow H_{2}, u \pi_{2}(x) u^{*}=$ 
$\pi_{1}(x)$ for all $x$ then $u \pi_{2}(g x) u^{*}=\pi_{1}(g x)$ for all $x$. Thus $\hat{g} \pi_{1}, \hat{g} \pi_{2}$ are equivalent. Thus every $g$ in Aut $\mathcal{M}$ acts on $\hat{\mathcal{M}}$ (where unitarily equivalent representations are identified).

If $E$ is a set of pure states, denote by $\hat{E}$ the subset of $\hat{\mathcal{M}}$ given by $\hat{E}=$ $\left\{\pi_{\phi} ; \phi \in E\right\}^{\wedge}=\left\{\pi_{\phi} ; \phi \in E\right\} / \sim$. If $g \in$ Aut $\mathcal{M}$ and $E$ is a set of pure states, then the equality $\hat{g} \hat{E}=\hat{E}$ will mean that $\left\{\pi_{\phi} ; \phi \in E\right\}^{\wedge}=\left\{\pi_{\phi} \circ g ; \phi \in E\right\}^{\wedge}$ (i.e. equality of sets in $\hat{\mathcal{M}}$ ).

If $\mathcal{M}$ is abelian then $\hat{\mathcal{M}}$ coincides with the set of all multiplicative states on $\mathcal{M}$. In general $\phi \rightarrow \pi_{\phi}$ will be a many-to-one map when $\phi$ ranges over the pure states of $\mathcal{M}$.

When no ambiguity arises we write equality when we mean $\sim . B(H)$ will denote the bounded linear operators on the Hilbert space $H$. The semigroup $S$ is left (right) amenable if there is a left (right) invariant state (or mean) (see M. M. Day [6] for more on this topic). A convex set $K$ of a Banach space $X$ has the (weak) Radon-Nikodým property (WRNP) RNP if for any finite measure space $(X B \mu)$ any countably additive $\mu$-continous map $m: B \rightarrow X$, such that $\mu(A)^{-1} m(A) \in K$ if $\mu(A) \neq 0$, is represented by a Bochner (Pettis) integrable function. For RNP (WRNP) sets see Stegall [16] (E. Saab [15]).

\section{Main results.}

THEOREM 1. Let $\mathcal{M}$ be a $W^{*}$ algebra and $G \subset$ Aut $\mathcal{M}$ a group of automorphisms $g: \mathcal{M} \rightarrow \mathcal{M}$. Let $E$ be a set of states on $\mathcal{M}$ such that $G^{*} E \subset E$ and denote $S_{E}^{G}=$ $\left\{\phi \in w^{*} \operatorname{cl} \operatorname{Co} E ; G^{*} \phi=\phi\right\}$.

(a) If $G$ is countable and amenable and $\phi_{0} \in w^{*} G_{\delta}\left(S_{E}^{G}\right)$ then there is a countable set $E_{0} \subset E$ such that $\pi_{\phi_{0}} \leq\left\{\bigoplus \pi_{\phi} ; \phi \in E_{0}\right\}$ and $\pi_{\phi_{0}}, \pi_{\phi}$ are not disjoint (see Pedersen $[12,3.8 .12])$ for each $\phi$ in $E_{0}$.

(b) If $S$ is any nonamenable group then there is some nonatomic probability space $(X B \mu)$ on which $S$ acts ergodically as measure preserving transformations such that if $E$ is the set of all pure states on $\mathcal{M}=L^{\infty}(X)$ then $S_{E}^{G}=w^{*} G_{\delta}\left(S_{E}^{G}\right)=\{\mu\}$, yet $\pi_{\mu}$ is not contained in $\left\{\bigoplus \pi_{\phi} ; \phi \in E\right\}$.

REMARKS. (i) Note that $E$ need not be $w^{*}$ closed but only $G^{*}$ invariant.

(ii) It is enough in (a) that $G$ be only a right amenable semigroup.

(iii) We show in (a) that there is some $h_{0} \in\left\{\bigoplus H_{\phi} ; \phi \in E_{0}\right\}$ such that $\phi_{0}(x)=$ $\left\langle\pi(x) h_{0}, h_{0}\right\rangle$ for all $x$ in $\mathcal{M}$, where $\pi=\left\{\bigoplus \pi_{\phi} ; \phi \in E_{0}\right\}$.

ProOF. Let $G$ be right amenable. Then the semigroup $G^{*}$ is left amenable. By our Theorem 1 in $[9] G^{*}$ has the $w^{*} G_{\delta}$ sequential property. Thus $\phi_{0}=w^{*} \lim \phi_{n}$ where $\phi_{n}$ is a sequence in CoE. A result of Akemann, Dodds, and Gamelin [1] implies that even $w \lim _{n} \phi_{n}=\phi_{0}$, where $w=\sigma\left(\mathcal{M}^{*}, \mathcal{M}^{* *}\right)\left(\right.$ while $\left.w^{*}=\sigma\left(\mathcal{M}^{*}, \mathcal{M}\right)\right)$. It follows then that there exists a sequence $\psi_{n}$ in $\operatorname{Co}\left\{\phi_{n}\right\} \subset \operatorname{Co} E$ such that $\| \psi_{n}-$ $\phi_{0} \| \rightarrow 0$. Each $\psi_{n}$ is a convex combination of a finite subset $E_{n}$ of $E$. Let $E_{0}=\bigcup_{1}^{\infty} E_{n}$. Thus if $\phi$ appears as a component in several $\psi_{n}$ 's, it appears in $E_{0}$ only once.

Let $\pi=\left\{\bigoplus \pi_{\phi} ; \phi \in E_{0}\right\}$ where $\left(\pi_{\phi}, H_{\phi}, h_{\phi}\right)$ is the cyclic representation induced by $\phi$. Each $\psi_{n}$ is a vector state on the $C^{*}$ algebra $\pi(\mathcal{M})$ (see [12, (1.5.7)]) acting on the Hilbert space $H=\left\{\bigoplus H_{\phi} ; \phi \in E_{0}\right\}$, i.e. $\psi_{n}(x)=\left\langle\pi(x) g_{n}, g_{n}\right\rangle$ for some $g_{n}$ in $H$ (see Remark 1). Furthermore $\phi_{0}(x)$ is a state on the $C^{*}$ algebra $\pi(\mathcal{M})$ since 
if $\pi x=0$, then $\phi(x)=0$ for all $\phi$ in $E_{0}$; hence $\psi_{n}(x)=0$ for all $n$, and $\phi_{0}(x)=0$. But we claim that $\left\|\psi_{n}-\phi_{0}\right\| \rightarrow 0$ in the norm of $\pi(\mathcal{M})$. This is readily implied by the fact that $\pi$ is an open map since $\pi(\mathcal{M})$ is a $C^{*}$ algebra $[\mathbf{1 2}, 1.5 .7]$.

We now apply Theorem D of R. Kadison [10, p. 307] and get that $\phi_{0}$ is also a vector state on $\pi(\mathcal{M})$ acting on $H$, i.e. there is some $h_{0} \in H$ such that $\phi_{0}(x)=$ $\left\langle\pi(x) h_{0}, h_{0}\right\rangle$.

If $\left\langle\pi_{\phi_{0}} H_{\phi_{0}} h_{\phi_{0}}\right\rangle$ is the cyclic corresponding to the state $\phi_{0}$ on $\mathcal{M}$ then $\phi_{0}(x)=$ $\left\langle\pi_{\phi_{0}}(x) h_{\phi_{0}}, h_{\phi_{0}}\right\rangle=\left\langle\pi(x) h_{0}, h_{0}\right\rangle$. It follows by Proposition 3.3.7 of [12] that $\pi_{\phi_{0}}$ is unitarily equivalent to $\pi$ restricted to $\left[\pi(\mathcal{M}) h_{0}\right] \subset H$. Hence $\pi_{\phi_{0}} \leq\left\{\bigoplus \pi_{\phi} ; \phi \in\right.$ $\left.E_{0}\right\}$. Let $E_{0}=\left\{\eta_{n} ; n=1,2, \ldots\right\}$ and $\left(\pi_{n}, H_{n}, h_{n}\right)=\left(\pi_{\eta_{n}}, H_{\eta_{n}}, h_{\eta_{n}}\right)$. Then there are $v_{n} \in H_{n}$ such that $h_{0}=\sum_{h} v_{n}, 1=\left\|h_{0}\right\|^{2}=\sum_{h}\left\|v_{n}\right\|^{2}$, and $\phi_{0}(x)=$ $\left\langle\pi(x) h_{0}, h_{0}\right\rangle=\sum_{n}\left\langle\pi(x) v_{n}, v_{n}\right\rangle=\sum_{n}\left\langle\pi_{n}(x) v_{n}, v_{n}\right\rangle$. Discard now from $E_{0}$ all $\eta_{n}$ 's for which the state $\left\langle\pi_{n}(x) v_{n}, v_{n}\right\rangle$ is 0 on $\pi_{n}(\mathcal{M})$ and let $E_{0}$ stand for the new set. If $\gamma_{n}(x)=\left\langle\pi_{n}(x) v_{n}, v_{n}\right\rangle=\left(\pi(x) v_{n}, v_{n}\right\rangle$ then $\gamma_{n}(x) \leq \phi_{0}(x)$ if $x \geq 0$ hence $\pi_{\gamma_{n}} \leq \pi_{\phi_{0}}$ by Pedersen [12, (3.3.8)] and $\pi_{\gamma_{n}} \leq \pi_{\eta_{n}}$ since $\pi_{\gamma_{n}}$ is unitarily equivalent to $\pi_{n}$ restricted to $\left[\pi_{n}(\mathcal{M}) v_{n}\right][\mathbf{1 2}, 3.3 .7]$. This set $E_{0}$ will satisfy part (a) of the theorem.

(b) If $G$ is any nonamenable group, there is by a result of Losert and Rindler [11] and J. Rosenblatt [14] a nonatomic probability space $(X, B, \mu)$ on which $G$ acts ergodically as a group of measure preserving transformations $g: X \rightarrow X$ such that there exists a unique $G$-invariant state on $L^{\infty}(X)$, which is necessarily $\mu$. Let $E$ be the set of all pure states on $L^{\infty}(X)=\mathcal{M}$. Then $S_{E}^{G}=\{\mu\}=w^{*} G_{\delta}\left(S_{E}^{G}\right)$. Assume now that $\pi_{\mu} \leq\left\{\bigoplus \pi_{\phi} ; \phi \in E\right\}$. Then, since each $\pi_{\phi}$ is one dimensional there are $\beta_{n}>0, \sum \beta_{n}=1$ such that $\mu(f)=\int f d \mu=\sum_{n=1}^{\infty} \beta_{n} \phi_{n}(f)$ for each $f$ in $\mathcal{M}$ for some multiplicative $\phi_{n}$ in $E$. But then our argument on p. 112 of [9] shows that the measure $\mu$ has to contain atoms, which cannot be.

REMARKS. (1) Let $\phi_{1}, \ldots, \phi_{m}$ be states on the $C^{*}$ algebra $A$ and $\psi=\sum_{1}^{m} \alpha_{i} \phi_{i}$ with $\alpha_{i}>0$ and $\sum_{1}^{m} \alpha_{i}=1$. Then $\pi_{\psi} \leq \bigoplus_{1}^{m} \pi_{\phi_{i}}$ : let $\left(\pi_{i}, H_{i}, h_{i}\right)=\left(\pi_{\phi_{i}}, H_{\phi_{i}}, h_{\phi_{i}}\right)$, then $\phi_{i}(x)=\left\langle\pi_{i}(x) h_{i}, h_{i}\right\rangle$. If $h=\sum_{1}^{m} \sqrt{\alpha_{i}} h_{i}$ then since $\pi(x) H_{i} \subset H_{i}$ we have

$$
\begin{aligned}
\langle\pi(x) h, h\rangle & =\sum \sqrt{\alpha_{i} \alpha_{j}}\left\langle\pi_{i}(x) h_{i}, h_{j}\right\rangle \\
& =\sum \alpha_{i}\left\langle\pi_{i}(x) h_{i}, h_{i}\right\rangle=\psi(x) .
\end{aligned}
$$

Thus $\pi_{\psi} \leq \bigoplus_{1}^{m} \pi_{i}$.

(2) With the notations of part (a) of the theorem let $E$ (hence $E_{0}=\left\{\eta_{n}\right\}$ ) consist only of pure states. Thus $\pi_{n}=\pi_{\eta_{n}}$ are irreducible. Since $\gamma_{n}(x)=\left\langle\pi_{n}(x) v_{n}, v_{n}\right\rangle$ are nonzero and $\pi_{\gamma_{n}} \leq \pi_{n}$ we get that $\pi_{n} \leq \pi_{\phi_{0}} \leq\left\{\bigoplus \pi_{k} ; k \geq 1\right\}$ for all $n$, where $\phi_{0}(x)=\sum \gamma_{n}(x)$ and $\sum\left\|v_{n}\right\|^{2}=1$.

Choose a maximal subsequence $\left\{\pi_{n_{i}}\right\} \subset\left\{\pi_{n}\right\}$ (possibly finite) such that $\pi_{n_{i}}, \pi_{n_{j}}$ are not unitarily equivalent if $i \neq j$. If $\pi_{n} \sim \pi_{n_{i}}$ there is some $v_{n}^{1} \in H_{n_{i}}$ such that $\gamma_{n}(x)=\left\langle\pi_{n_{i}}(x) v_{n}^{1}, v_{n}^{1}\right\rangle$ and $\left\|v_{n}\right\|=\left\|v_{n}^{1}\right\|$. Hence if $\varepsilon_{i}(x)=\left\{\sum \gamma_{n}(x) ; \pi_{n} \sim \pi_{n_{i}}\right\}$ then $\varepsilon_{i}(x)=\sum_{k}\left\langle\pi_{n_{i}}(x) v_{k}^{i}, v_{k}^{i}\right\rangle$ for some sequence $v_{k}^{i} \in H_{n_{i}}$ with $\sum_{k}\left\|v_{k}^{i}\right\|^{2}<\infty$. Thus $\varepsilon_{i} \neq 0$ can be considered as a positive normal functional on $\left(\pi_{n_{i}}, H_{n_{i}} h_{n_{i}}\right)$ and $\varepsilon_{i}(1)=\sum_{k}\left\|v_{k}^{i}\right\|^{2} \neq 0, \phi_{0}(x)=\sum_{i} \varepsilon_{i}(x)$, and $\phi_{0}(1)=1=\sum_{i} \varepsilon_{i}(1)$. 
Now let $Z_{0}=\left\{z_{\alpha} ; \alpha \in I\right\}$ be the set of all minimal central projections of the second dual $\mathcal{M}^{* *}$ of $\mathcal{M}^{1}$ For each $g$ in $G, g^{* *}$ is an automorphism on $\mathcal{M}^{* *}[\mathbf{1 2}$, (7.4.5), p. 244] and hence $g^{* *}: Z_{0} \rightarrow Z_{0}$, one-to-one onto. Since $z_{\alpha} z_{\beta}=0$ unless $\alpha=\beta,\left(g^{* *} z_{\alpha}\right)\left(g^{* *} z_{\beta}\right)=0$ unless $\alpha=\beta$ (see Dixmier [7, 5.2.4-5.2.8, p. 103]).

Let $z_{\alpha_{i}} \in Z_{0}$ be the central support of $\pi_{n_{i}}$. Then since $\varepsilon_{i}$ is $\pi_{n_{i}}$-normal and is also in $\mathcal{M}^{*}$ we have $\varepsilon_{i}\left(x z_{\alpha_{i}}\right)=\varepsilon_{i}(x)$ for all $x$ in $\mathcal{M}$ (where we consider $\mathcal{M} \subset \mathcal{M}^{* *}$ ) and $\varepsilon_{i}\left(x z_{\alpha}\right)=0$ if $\alpha \in I$ and $\alpha \neq \alpha_{i}$ for all $x$ in $\mathcal{M}$ (see Takesaki [17, p. 125] and Akemann and Shulz [2, Proposition A1, p. 110 and Proposition A10, p. 116]). Now denote $\beta_{j}=\varepsilon_{j}(1)=\varepsilon_{j}\left(z_{\alpha_{j}}\right)$ and fix some $\beta_{i}$. Let $F=\left\{\alpha_{j} ; \beta_{j}=\beta_{i}\right\}$. Clearly $F$ is finite since $\sum \beta_{j}=1$. Let $g \in G$, then $g^{* *} z_{\alpha_{i}}=z_{\delta}$ for some $\delta$ in $I$. But

$$
\phi_{0}\left(z_{\alpha_{i}}\right)=\sum_{j} \varepsilon_{j}\left(z_{\alpha_{i}}\right)=\varepsilon_{i}\left(z_{\alpha_{i}}\right)=\beta_{i}=\phi_{0}\left(g^{* *} z_{\alpha_{i}}\right)=\phi_{0}\left(z_{\delta}\right)=\sum_{j} \varepsilon_{j}\left(z_{\delta}\right) .
$$

But $\varepsilon_{j}\left(z_{\delta}\right) \neq 0$ for at most one $j$ and since $\beta_{i} \neq 0$ there is such a $j$ (see [7, 5.2.8]). Thus $g^{* *} z_{\delta}=z_{\alpha_{k}}, \varepsilon_{k}\left(z_{\alpha_{k}}\right)=\beta_{k}=\beta_{i}$ for some $k$, and thus $\alpha_{k} \in F$. If $Z_{F}=\left\{z_{\alpha_{j}} ; \alpha_{j} \in F\right\}$ then we have shown that $g^{* *} Z_{F} \subset Z_{F}$ and since $Z_{F}$ is finite and $g^{* *}$ is one-to-one, $g^{* *} Z_{F}=Z_{F}$ for all $g$ in $G$. This however implies that $\left\{\pi_{n_{j}} \circ g ; \alpha_{j} \in F\right\}=\left\{\pi_{n_{j}} ; \alpha_{j} \in F\right\}$ for all $g$, since $\pi_{n_{j}}$ and $\pi_{n_{j}} \circ g$ are irreducible (see $[12,(3.8 .12),(3.13 .3)])$.

Now let $\beta_{i_{k}}$ be a maximal set of different $\beta_{i}$ 's. For each $k$ let $F_{k}=\left\{\alpha_{j} ; \beta_{j}=\beta_{i_{k}}\right\}$.

Let $R_{k}=\left\{\pi_{n_{j}} ; \beta_{j}=\beta_{i_{k}}\right\}$. Then the $R_{k}$ are finite, pairwise disjoint sets such that $\bigcup_{k} R_{k}=\left\{\pi_{n_{i}} ; i=1,2, \ldots\right\}$. Furthermore for each $g$ in $G$ and $k,\left\{\pi_{n_{j}}\right.$ 。 $\left.g ; \pi_{n_{j}} \in R_{k}\right\}=R_{k}$ (up to unitary equivalence). Each finite set $R_{k}$ can be further partitioned into finitely many minimal $G$-invariant sets $R_{j}^{k}$, i.e. subsets such that $\{\rho \circ g ; g \in G\}=R_{j}^{k}$ for each $\rho$ in $R_{j}^{k}$ and $\left\{\rho \circ g ; \rho \in R_{j}^{k}\right\}=R_{j}^{k}$ for each $g$ in $G$ (all above equalities are up to unitary equivalence). We thus have

COROLLARY 1. (a) Let $G$ be a group of automorphisms on the $W^{*}$ algebra $\mathcal{M}$ and $E$ a set of pure states on $\mathcal{M}$ such that $G^{*} E \subset E$ and let $S_{E}^{G}=\{\phi \in$ $\left.w^{*} \operatorname{clCo} E ; G^{*} \phi=\phi\right\}$. Assume that $\phi_{0} \in w^{*} G_{\delta}\left(S_{E}^{G}\right)$. that

If $G$ is countable and amenable then there is a countable subset $E_{0} \subset E$ such

$$
\pi_{\psi} \leq \pi_{\phi_{0}} \leq\left\{\bigoplus \pi_{\phi} ; \phi \in E_{0}\right\} \quad \text { for all } \psi \in E_{0} \text { (by Theorem } 1(\mathrm{a}) \text { ). }
$$

Furthermore $\left\{\pi_{\phi} ; \phi \in E_{0}\right\}^{\wedge}=\hat{E}_{0}=\bigcup_{k} R_{k}$ is a countable (or finite) disjoint union of finite minimal $G$ invariant sets $R_{k} \subset \hat{\mathcal{M}}$, i.e. the finite sets $R_{k}$ satisfy $R_{k} \cap R_{j}=\varnothing$ if $k \neq j, \hat{g} R_{k}=R_{k}$, and $\{\rho \circ g ; g \in G\}=R_{k}$ for all $g$ in $G, \rho$ in $R_{k}$ and all $k$.

(b) No nonamenable group has the above "strong containment property" as part (b) of the previous theorem shows.

REMARKS. (1) $G$ need only be a right amenable semigroup (as in Day [6]).

(2) Fix $k$ and define in $G$ the equivalence relation $g_{1} \sim g_{2}$ iff $\rho \circ g_{1}=\rho \circ g_{2}$ (are unitarily equivalent) for all $\rho$ in $R_{k}$. Then $G$ modulo $\sim$ becomes a semigroup $G_{k}$ of one-to-one maps on the finite set $R_{k}$. Thus $G_{k}$ is a finite group and, if $G$

${ }^{1}$ We acknowledge with thanks communications we had with Alan L. T. Paterson. The proof below and the statement of Corollary 1 are different than the ones suggested in these inspiring communications. 
is a group, for each $k$, card $R_{k}$ is the cardinality of a finite coset $G / H_{k}$ for some subgroup $H_{k} \subset G$.

If $J \subset \mathcal{M}$ denote $J^{0}=\left\{\phi \in \mathcal{M}^{*} ;\langle\phi, f\rangle=0\right.$ for all $f$ in $\left.J\right\}$.

COROLLARY 2. Let $G \subset$ Aut $\mathcal{M}$ be a semigroup and $E$ a set of pure states on M. Assume that for some countable $J \subset \mathcal{M}, S_{E}^{G} \cap J^{0}$ is nonvoid and has the $R N P$.

(a) If $G$ is countable and amenable then every subset $E_{1} \subset w^{*} \operatorname{cl} E$ such that $G^{*} E_{1} \subset E_{1}$ and $S_{E_{1}}^{G} \cap J^{0} \neq \varnothing$ contains a finite subset $E_{0} \subset E_{1}$ such that $\hat{g} \hat{E}_{0}=\hat{E}_{0}$ for all $g$ in $G$ and $\left\{\pi_{\phi} \circ g ; g \in G\right\}=\hat{E}_{0}$ for all $\phi$ in $E_{0}$ (i.e. $\hat{E}_{0}$ is a finite minimal $G$ invariant subset of $\left.\hat{E}_{1}\right)$.

(b) If $G$ is any nonamenable group then the ergodic measure preserving action of $G$ on $L^{\infty}(X)=\mathcal{M}$, for the nonatomic probability space $(X B \mu)$ of Theorem $1(\mathrm{~b})$, satisfies $S^{G}=\{\mu\}$ and has the $R N P$, yet for no finite set $E_{0}$ of pure states on $\mathcal{M}$ does $\hat{g} \hat{E}_{0} \subset \hat{E}_{0}$, for all $g$ in $G$ (equivalently $G^{*} E_{0} \subset E_{0}$ ), hold true.

REMARKS. (i) $w$ compact or norm separable $w^{*}$ compact convex sets have the RNP (Stegall [16, Proposition 1.10]). (ii) $G$ need only be right amenable. (iii) $S_{E_{1}}^{G} \neq \varnothing$ by the Markov-Kakutani-Day fixed point theorem, however $S_{E_{1}}^{G} \cap J^{0} \neq \varnothing$ may not hold, hence we need to assume it.

ProOF. (a) If $F=w^{*} \operatorname{cl} E$ then $S_{E}^{G}=S_{F}^{G}$; hence we can assume that $E$ is $w^{*}$ closed. Now subsets of closed bounded RNP sets have the RNP [16, p. 508]; hence $S_{E_{1}}^{G} \cap J^{0} \neq \varnothing$ has the RNP. Since $S_{E_{1}}^{G} \cap J^{0}$ is $w^{*}$ compact and has the RNP it necessarily has a $w^{*} G_{\delta}$ point $\phi_{0}$ (see [16] or for alternate proof see $[\mathbf{9}, \mathrm{p} .116]$ ) which is necessarily a $w^{*} G_{\delta}$ point of $S_{E}^{G}$, since $J$ is countable. Corollary 1 (a) finishes the proof.

(b) The easy proof is left for the reader (or see remark (a) on p. 115 of $[9]$ ).

If we assume that $\mathcal{M}$ is abelian then we get the following improvement (in a sense) of our Theorem 4 of [9]:

COROLlaRY 3. (a) Let $\mathcal{M}$ be an abelian $W^{*}$ algebra, i.e. $\mathcal{M}=L^{\infty}(\Gamma, \mu)$ for some locally compact $\Gamma$ and positive Radon measure $\mu[17$, Theorem $1.18, p .119]$.

Let $E$ be a set of multiplicative states on $\mathcal{M}$ and $G \subset$ Aut $\mathcal{M}$ a countable (right) amenable semigroup such that the nonvoid set $S_{E}^{G} \cap J^{0}$ has the RNP for some countable $J \subset \mathcal{M}$. If $E_{1} \subset w^{*} \mathrm{cl} E$ is any set such that $G^{*} E_{1} \subset E_{1}$ and $S_{E_{1}}^{G} \cap J^{0} \neq \varnothing$ then $E_{1}$ contains a finite subset $E_{0}$ such that $G^{*} E_{0} \subset E_{0}$.

(b) No nonamenable group has the above "RNP-finite invariant property" (by Corollary 2(b)).

REMARKS. (1) If $\phi_{0} \in S_{E}^{G} \cap J^{0}$ then the set $F=\operatorname{supp} \phi_{0}$ is a $w^{*}$ closed subset of $E$ such that $G^{*} F \subset F$ (where supp $\phi_{0}$ is the smallest $w^{*}$ closed set $E^{\prime} \subset E$ such that $\left.\phi_{0} \in w^{*} \mathrm{cl} \mathrm{Co} E^{\prime}\right)$.

(2) If $J=\{0\}$ then $S_{E}^{G}$ need only have WRNP in order that Corollary 3(a) hold (see $[9$, Corollary 6]). We improve this in the next corollary.

If we take $J=\{0\}$ and impose certain restrictions on $E$ and on the action of $G$ on $\mathcal{M}$ then we can replace the RNP by the WRNP:

COROLlaRY 4. (a) Let $G \subset$ Aut $\mathcal{M}$ be a group such that $S^{G}$ is a simplex. Let $E$ be a set of pure states on $\mathcal{M}$ such that $u^{*} \phi u \in E$ for each unitary $u$ in $\mathcal{M}$ and $\phi \in E$. Assume that $S_{E}^{G} \neq \varnothing$ and has the WRNP. 
If $G$ is countable and amenable then every set $E_{1} \subset w^{*} \mathrm{cl} E$ such that $G^{*} E_{1} \subset E_{1}$ contains a finite subset $E_{0}$ such that $\hat{g} \hat{E}_{0}=\hat{E}_{0}$ and $\left\{\hat{g} \pi_{\phi} ; g \in G\right\}=\hat{E}_{0}$ for all $g$ in $G$ and $\phi$ in $E_{0}$ (i.e. such that $\hat{E}_{0}$ is finite minimal $G$ invariant).

(b) No nonamenable group has the above property (by Corollary 2(b)).

Proof. Since $S^{G}$ is a simplex the positive cone $C^{G}=\left\{\bigcup \lambda S^{G} ; \lambda \geq 0\right\}$ is a lattice, i.e. $\phi_{1}, \phi_{2} \in C^{G}$ implies that $\phi_{1} \wedge \phi_{2}$ exists and is in $C^{G}$ (see Asimov and Ellis $\left[3\right.$, pp. 49, 70]). We claim that $C_{E}^{G}=\left\{\bigcup \lambda S_{E}^{G} ; \lambda \geq 0\right\}$ is also a lattice. In fact let $\phi_{1}, \phi_{2} \in C_{E}^{G}$ and $\phi_{0}=\phi_{1} \wedge \phi_{2} \in C^{G}$. We show that $\phi_{0} \in C_{E}^{G}$. Clearly $\phi_{0} \leq \phi_{1}$, hence by $[12,3.3 .8], \pi_{\phi_{0}} \leq \pi_{\phi_{1}}$, i.e. $\phi_{0}(x)=\left\langle\pi_{\phi_{1}}(x) \xi_{1}, \xi_{1}\right\rangle$ for some $\xi_{1} \in H_{\phi_{1}}$. Now for all $x, \phi_{1}(x)=\lim \phi_{\alpha}(x)$ where $\phi_{\alpha} \in \operatorname{Co} \lambda_{1} E$ where $\lambda_{1}=\left\|\phi_{1}\right\|$. Also, if $\pi_{\mu}(x)=0$ for all $\mu \in E$ then $\pi_{\mu}\left(x^{*} x\right)=0$ for all $\mu \in E$; thus $\mu\left(x^{*} x\right)=0$ for all $\mu \in E$ hence $\phi_{1}\left(x^{*} x\right)=0$ which implies, since $\phi_{0} \leq \phi_{1}$, that $\phi_{0}\left(x^{*} x\right)=0$. Thus by Cauchy-Schwarz, $\phi_{0}(x)=0$. Hence $\phi_{0}=0$ on $\left\{\bigcap \operatorname{Ker} \pi_{\mu} ; \mu \in E\right\}$. If $\phi_{0}=0$ then $\phi_{0} \in C_{E}^{G}$. If $\phi_{0} \neq 0$ we can assume that $\phi_{0}$ is a state. Then $\phi_{0}$ is a $w^{*}$ limit of positive elements of type $\sum_{1}^{n}\left\langle\pi_{\mu_{i}}(x) \xi_{i}, \xi_{i}\right\rangle=\nu(x)$ with $\nu(1)=1$ for some $\xi_{i}$ in $H_{\mu_{i}}$ and $\mu_{i}$ in $E$, by Dixmier $[7,3.4 .2$ and 3.4.4, p. 66].

We now claim that $x \rightarrow\left\langle\pi_{\mu}(x) \xi_{0} \xi_{0}\right\rangle=\mu_{0}(x)$ belongs to $w^{*} \operatorname{cl} E$ for each $\mu \in E$ and $\xi_{0} \in H_{\mu}$ such that $\left\|\xi_{0}\right\|=1$. In fact there is a unitary $u \in B\left(H_{\mu}\right)$ such that $u \xi_{\mu}=\xi_{0}$ and there is a net of unitaries $u_{\alpha} \in \mathcal{M}$ such that $\pi_{\mu}\left(u_{\alpha}\right) \rightarrow u$ strongly on $H_{\mu}$ by Kaplanski's density theorem $[\mathbf{1 2}, 2.3 .3]$ and since $\pi_{\mu}$ is irreducible. But then $\pi_{\mu}(x) \pi_{\mu}\left(u_{\alpha}\right) \xi_{\mu} \rightarrow \pi_{\mu}(x) \xi_{0}$ in norm. Thus for all $x$ in $\mathcal{M},\left\langle\pi_{\mu}\left(u_{\alpha}^{*} x u_{\alpha}\right) \xi_{\mu}, \xi_{\mu}\right\rangle \rightarrow$ $\left\langle\pi_{\mu}(x) \xi_{0}, \xi_{0}\right\rangle$ as readily seen. (If $\left\|\xi_{\alpha}-\xi\right\| \rightarrow 0$ then for all bounded $T,\left\langle T \xi_{\alpha}, \xi_{\alpha}\right\rangle \rightarrow$ $\langle T \xi, \xi\rangle$.) It follows then that $\phi_{0}$ is a $w^{*}$ limit of a net in $\operatorname{Co}\left\{w^{*} \operatorname{cl} E\right\}$ hence $\phi_{0} \in$ $S^{G} \cap w^{*} \operatorname{cl}$ Co $E$ which by definition is $S_{E}^{G}$. We have shown that for all $\phi_{1}, \phi_{2} \in$ $C_{E}^{G}, \phi_{1} \wedge \phi_{2}$ exists and is in $C_{E}^{G}$. But then $C_{E}^{G}-C_{E}^{G}$ (the linear span of $C_{E}^{G}$ ) is a lattice [3, p. 49]). But then $C_{E}^{G}-G_{E}^{G}$, over the reals, is an abstract $L$ space [3, p. 70, Theorem 7.1 and p. 14, Theorem 4.7]. Now $S_{E}^{G}-S_{E}^{G}$ has the WRNP by Saab [15, Theorem 1(i)]. However this last set is just the closed unit ball of the abstract $L$ space $C_{E}^{G}-C_{E}^{G}$. Thus the Banach lattice $C_{E}^{G}-C_{E}^{G}$ has the WRNP. We now apply Proposition 8 of Ghoussoub and Saab [8] and get that $C_{E}^{G}-C_{E}^{G}$ even has the RNP. and hence so does (each bounded subset) $S_{E}^{G}$. Now apply Corollary 2(a) with $J=\{0\}$.

REMARKS. $S^{G}$ is a simplex at least in the case when $(\mathcal{M}, G, \alpha)$ is weakly asymptotically abelian (Pedersen $[\mathbf{1 2}, 7.13 .1]$ or even if $\alpha_{G}$ is a large group of automorphisms of $\mathcal{M}\left[12,7.12 .5\right.$ and 7.13.2]). However $S^{G}$ may be a simplex, yet $\alpha_{G}$ need not be a large group of automorphisms (Takesaki $\left[17\right.$, pp. 252-253]). $S^{G}$ is a simplex if and only if the pair $(\mathcal{M}, \phi)$ is $G$-abelian (Bratteli-Robinson [4, Definition 4.3.6, p. 374]) for each $\phi \in S^{G}$, iff $E_{\phi} \pi_{\phi}(\mathcal{M}) E_{\phi}$ is abelian for all $\phi \in S^{G}$ where $E_{\phi}$ is the projection from $H_{\phi}$ to $\left\{\xi \in H_{\phi} ; u_{\phi}(g) \xi=\xi\right.$ for all $g$ in $\left.G\right\}$ (see [4, Corollary 4.3.11, p. 379]).

THE CASE WHERE $E \subset \mathcal{M}_{*}$. If $E$ is a set of normal states on $\mathcal{M}$ then $G$ need not be amenable and $G^{*} E \subset E$ need not hold in Theorem 1 and its corollaries. Stronger results are available in this case by adapting the results in [19] to the $W^{*}$ algebra context. If $K \subset \mathcal{M}^{*}, w^{*} \operatorname{seq} \mathrm{cl} K$ denotes the $w^{*}$ sequential closure of $K$. $\mathcal{M}_{*}$ is considered as embedded in $\mathcal{M}^{*}$. 
THEOREM 2. Let $\mathcal{M}$ be $a W^{*}$ algebra and $G \subset$ Aut $\mathcal{M}$ a countable set. Let $E \subset \mathcal{M}_{*}$ be a set of (normal) states and $S_{E}^{G}=\left\{\phi \in w^{*} \operatorname{cl}\right.$ Co $\left.E ; G^{*} \phi=\phi\right\}$.

If $\phi_{0} \in w^{*} G_{\delta}\left(S_{E}^{G}\right)$ then $\phi_{0} \in \operatorname{norm} \operatorname{clCo} E \subset \mathcal{M}_{*}$ and $\pi_{\phi_{0}} \leq\left\{\bigoplus \pi_{\phi} ; \phi \in E_{0}\right\}$ for some countable set $E_{0} \subset E$.

Furthermore, if for some countable $J \subset \mathcal{M}, S_{E}^{G} \cap J^{0}$ has the WRNP or card $S_{E}^{G} \cap$ $J^{0}<2^{c}$ and in fact if $S_{E}^{G} \cap J^{0}$ does not contain $a$ " $w^{*}$ affine isomorph" of the "big" set $\mathcal{F}=\left\{\phi \in S_{l \infty} ; \phi(f)=0\right.$ for $f$ in $\left.c_{0}\right\} \subset\left(l^{\infty}\right)^{*}$ then there is some $\phi_{0}$ in $S_{E}^{G} \cap J^{0} \cap$ norm cl Co $E \subset \mathcal{M}_{*}$ such that $\pi_{\phi_{0}} \leq\left\{\bigoplus \pi_{\phi} ; \phi \in E_{0}\right\}$ for some countable $E_{0} \subset E$.

REMARKS. $\quad c_{0}=\left\{f \in l^{\infty} ; \lim _{n} f(n)=0\right\}$ and $S_{l \infty}$ is the set of states on $l^{\infty}$. By " $w^{*}$ affine isomorph" we mean by a $w^{*}-w^{*}$ continuous norm isomorphism into, $t^{*}: l^{\infty *} \rightarrow \mathcal{M}^{*}$, such that $t^{*} \mp \subset S_{E}^{G} \cap J^{0}$, as in [19, Theorem 1.4(b)]. Note that $\mathcal{F}=w^{*} \operatorname{cl} \operatorname{Co}(\beta N \sim N)$ and $\operatorname{card} \mathcal{F}=2^{c}$, where $c$ is the cardinality of the continuum, as well known. $G$ can be replaced by any set of $w^{*}$ continuous operators on $\mathcal{M}$. This theorem is false for uncountable $G$.

ProOF. Any $g$ in Aut $\mathcal{M}$ is $w^{*}$ continuous on $\mathcal{M}[17$, p. 135, Corollary 3.10]. It follows now that $\left\{\phi_{0}\right\} \subset w^{*}$ seq cl Co $E$ since if not then by Theorem 1.4(b) of [19] the set $\left\{\phi_{0}\right\}$ would contain a $w^{*}$ affine isomorph of $\mathcal{F}$, which cannot be. (We cannot apply our Theorem 1 here since $G$ need not be amenable.) Thus $\phi_{0}=w^{*} \lim \phi_{n}$ for some sequence $\phi_{n}$ in Co $E$. The rest of the proof is verbatim like that of Theorem 1.

If $S_{E}^{G} \cap J^{0}$ has the WRNP or $\operatorname{card}\left(S_{E}^{G} \cap J^{0}\right)<2^{c}$ then apply Corollary $1.4^{\prime}$ of [19] and get that there is some $\phi_{0}$ in $S_{E}^{G} \cap J^{0} \cap w^{*}$ seq cl Co $E$. But then, the proof of Theorem 1 shows that $\phi_{0} \in$ norm $\operatorname{clCo} E$ and $\pi_{\phi_{0}} \leq\left\{\bigoplus \pi_{\phi} ; \phi \in E_{0}\right\}$ for some countable set $E_{0} \subset E$.

If $S_{E}^{G} \cap J^{0}$ does not contain a " $w^{*}$ affine isomorph" of $₹$ then apply Theorem 1.4(b) of [19] and get that there is some $\phi_{0} \in S_{E}^{G} \cap J^{0} \cap w^{*}$ seq cl Co $E$. For the rest argue as above.

ADDED IN PROOF. We have obtained the following result using results in [19] (which in turn use in part techniques of Ching Chou):

THEOREM. Let $G \subset$ Aut $\mathcal{M}$ be a countable group and $E$ a set of pure states on $\mathcal{M}$ such that $G^{*} E \subset E$ and $J \subset \mathcal{M}$ countable.

(a) Assume that $G$ is amenable: (i) If $\phi \neq S_{E}^{G} \cap J^{0} \subset \mathcal{M}_{*}$ then $\mathcal{M}$ contains minimal projections (in the absence of such, $S^{G} \cap J^{0} \cap\left\{\mathcal{M}^{*} \sim \mathcal{M}_{*}\right\}$ contains $a$ " $w^{*}$ affine isomorph of the big set $\mathcal{F}^{\prime \prime)}$. (ii) $S^{G} \cap \mathcal{M}_{*}^{\perp}$ contains a " $w^{*}$ isomorph of the big set F").

$A$ fortiori no action of a countable amenable group on an infinite dimensional $W^{*}$ algebra has a unique $G$ invariant state on $\mathcal{M}$.

(b) If $G$ is any nonamenable group then the action of $G$ on $L^{\infty}(X)$ of Theorem 1(b) violates both (a)(i) and (a)(ii).

REMARKS. 1. $\mathcal{M}^{*} \sim \mathcal{M}_{*}$ is the set theoretical difference of $\mathcal{M}^{*}$ and $\mathcal{M}_{*} \subset \mathcal{M}^{*}$. $\mathcal{M}_{*}^{\perp}$ is the set of singular elements of $\mathcal{M}^{*}[17$, p. 127].

2. This theorem improves our Theorem 3 in [9] which in turn is a result of $K$. Schmidt [20] and J. Rosenblatt [14]. Our only assumption on $M$ is that it is infinite dimensional. 


\section{REFERENCES}

1. C. A. Akemann, P. G. Dodds, and J. L. B. Gamelin, Weak compactness in the dual space of a $C^{*}$ algebra, J. Funct. Anal. 10 (1972), 446-450.

2. C. A. Akemann and F. Schulz, Perfect $C^{*}$-algebras, Mem. Amer. Math. Soc. No. 326 (1985).

3. L. Asimov and A. J. Ellis, Convexity theory and its applications in functional analysis, Academic Press, New York, 1980.

4. Ola Bratteli and D. W. Robinson, Operator algebras and quantum statistical mechanics. I, Springer-Verlag, Berlin and New York, 1979.

5. Cho-Ho Chu, $A$ note on scattered $C^{*}$ algebras and the Radon Nikodym property, J. London Math. Soc. 24 (1981), 533-536.

6. M. M. Day, Amenable semigroups, Illinois J. Math. 1 (1957), 509-544.

7. J. Dixmier, Les $C^{*}$-algèbres et leurs représentations, 2nd ed., Gauthier-Villars, Paris, 1969.

8. N. Ghoussoub and E. Saab, On the weak Radon Nikodym property, Proc. Amer. Math. Soc. 81 (1981), 81-84.

9. E. E. Granirer, Geometric and topological properties of certain $w^{*}$ compact convex sets which arise from the study of invariant means, Canad. J. Math. 37 (1985), 107-121.

10. R. V. Kadison, States and repesentations, Trans. Amer. Math. Soc. 103 (1962), 304-319.

11. V. Losert and H. Rindler, Almost invariant sets, Bull. London Math. Soc. 13 (1981), 145148.

12. G. K. Pedersen, $C^{*}$-algebras and their automorphism groups, Academic Press, New York, 1979.

13. R. R. Phelps, Lectures on Choquet's theorem, Van Nostrand Math. Studies, No. 7, Van Nostrand, Princeton, N. J., 1966.

14. J. Rosenblatt, Uniqueness of invariant means for measure preserving transformations, Trans. Amer. Math. Soc. 265 (1981), 623-636.

15. E. Saab, Some characterisations of weak Radon Nikodym sets, Proc. Amer. Math. Soc. 86 (1982), 307-311.

16. C. Stegall, The Radon Nikodym property in conjugate Banach spaces. II, Trans. Amer. Math. Soc. 264 (1981), 507-519.

17. M. Takesaki, Theory of operator algebras. I, Springer-Verlag, Berlin and New York, 1979.

18. E. E. Granirer, Exposed points of convex sets and weak sequential convergence, Mem. Amer. Math. Soc. No. 123 (1972).

19. __ Geometric and topological properties of certain $w^{*}$ compact convex subsets of double duals of Banach spaces which arise from the study of invariant means, Illinois $\mathbf{J}$. Math. 30 (1986), 148-174.

20. K. Schmidt, Amenability, Kazhdan's property $T$, strong ergodicity and invariant means for ergodic group actions, Ergodic Theory Dynamical Systems 1 (1981), 223-236.

Department of MAThematics, The UNIVERsity of BRITISH Columbia, VANCOUVER, CANADA 